\title{
A Combination Algorithm of Multiple Lattice-Valued Concept Lattices
}

\author{
Li Yang \\ School of Mathematics and Information Science, North China University of Water Resources \\ and Electric Power, Zhengzhou 450046, Henan, PR China \\ Yuhui Wang \\ College of Traffic \& Transportation, Southwest Jiaotong University, \\ Chengdu 610031, Sichuan, PR China \\ Yang $\mathrm{Xu}$ \\ Intelligent Control Development Center, Southwest Jiaotong University, \\ Chengdu 610031, Sichuan, PR China \\ Received 21 June 2012
Accepted 9 March 2013
}

\begin{abstract}
With the rapid development of network technology, especially in the internet area, distributed computation and parallel processing of data are urgently needed. This paper studies the combination operation of the lattice-valued concept lattices to realize its distributed computation and parallel processing. The proposal of its corresponding combination algorithm is mainly according to the conjunction properties of incomparable attribute values. Firstly, we define a homotypic lattice-valued concept lattice and a combined concept lattice; secondly, we analyze some relations between the lattice-valued formal concepts under two conditions and prove the isomorphism of the combination theory; finally, we present the combination algorithm of multiple lattice-valued concept lattices and analyze the algorithm complexity and employ an example to show the application of this combination algorithm.
\end{abstract}

Keywords: Concept lattice; Lattice-valued concept lattice; Homotypic formal context; Combination algorithm; Parallel processing

\section{Introduction}

Formal concept analysis (FCA) was proposed by Wille in 1982, and its ideological core is to construct the binary relation between objects and attributes based on the bivalent logic [1-3]. The basic setting is well-suited for attributes which are crisp, i.e., each object of the domain of applicability of the attribute either has (1) or does not have (0) the attribute. However, in most cases, many attributes are fuzzy rather than crisp. That is to say, it is a matter of degree to which an object has a fuzzy attribute. For instance, when asking whether a man with a height of
$182 \mathrm{~cm}$ is tall, one probably gets an answer like "not absolutely tall but almost tall". If according to the fuzzy logic, we can say that a man with a height of $182 \mathrm{~cm}$ is tall to a degree, say, 0.8. So, the entries of a table describing objects and attributes become degrees from $[0,1]$. As a conceptual clustering method, concept lattices and fuzzy concept lattices have been applied in many fields, e.g., conceptual clustering method [4,5], information retrieval and knowledge discovery [6].

However, there is another way to characterize such fuzzy information. The way is based on the lattice implication algebra considering the modifiers "almost,

Corresponding author: School of Mathematics and Information Science, North China University of Water Resources and Electric Power, Zhengzhou 450046, Henan, PR China

E-mail addresses: yangli6672@sina.com 
rather, very, absolutely, etc." as its elements. A favorite choice of $L$ is the set of modifiers instead of values from $[0,1]$. Doing so, the entries of a table describing objects and attributes become the modifiers from $L$. Namely, one can consider a table with modifiers from $L$ a lattice-valued context and the structures which result this way are called the lattice-valued concept lattices and its related theories have been studied in our previous work [7-10]. The key point different from the general fuzzy concept lattices is that the range of values achieved directly by the lattice-valued concept lattice is not a unit interval $[0,1]$ but a complete lattice structure, on which both incomparability and fuzzy information can be dealt with very well.

Whether the classical and fuzzy concept lattices or the lattice-valued concept lattices, the prerequisite for their applications is to construct the appropriate structures. In the practical construction process, it is inevitable to perform the complex calculations, so that the high requirements put forward for precision and real time. Especially for a large scale formal context, the present algorithms of constructing concept lattices can not effectively decrease the time complexity. Under such situation, more and more researchers tend to use parallel and distributed store techniques to build the concept lattice, whose idea is to firstly decompose the formal context into many sub-contexts and construct the corresponding sub-lattices, then obtain the final concept lattice by combining these sub-lattices, i.e., the decomposition and combination operations on concept lattices. Through the decomposition operation, the formal context can be transformed from the more complex into relatively simpler, which can reduce the computation steps and improve the speed of construction; and through the combination operation, the finally complete concept lattice can be easily obtained by combining these simpler sub-lattices. As the core content about the combination algorithms, many horizontal combination algorithms and vertical combination algorithms of classical concept lattices have emerged [11-13, which indeed improved the construction efficiency.

However, the above on combination operations are not involved to the fuzzy concept lattice, the major reason is that, for quite a long time, people are used to transforming the fuzzy concept lattice into the classical concept lattice through the threshold values [14] designed for solving fuzzy problems, which limits the development of the fuzzy concept lattice. And due to the fact that the lattice-valued concept lattice has mathematical properties, it is inevitable to research the combination algorithms of multiple lattice-valued concept lattices, which is an effectively basic approach for researching the distributed computation and parallel processing of the lattice-valued concept lattice.

This paper presents a combination algorithm of multiple lattice-valued concept lattices according to the conjunction properties among attribute values derived from the lattice implication algebra. In Section 2, an overview of the classical concept lattice and the lattice implication algebra is given. In Section 3, the related works of the lattice-valued concept lattice are briefly summarized. The definitions of homotypic lattice-valued context and homotypic lattice-valued concept lattice are proposed in Section 4. The combined context and the combined concept lattice are defined. In order to merge the needs for combining the multiple lattice-valued concept lattices, we discuss the relationship between the different formal concepts generated respectively from the same and the different formal contexts in detail. In addition, the important conclusion is obtained: the lattice-valued concept lattice derived from the combination of multiple formal sub-contexts is proved isomorphic to the combination of multiple lattice-valued sub-lattices derived from these formal sub-contexts. Finally, the feasibility and effectiveness of this combination algorithm are analyzed and further demonstrated through an example. Concluding remarks are presented in Section 5 . 


\section{Concept lattice and lattice implication algebra}

In this section, we briefly review the classical concept lattices and the lattice implication algebra which are the foundations of the lattice-valued concept lattice.

Definition 2.1 ( [15] ) A partial ordered set is a set in which a binary relation $\leq$ is defined, which satisfies the following conditions: for any $x, y, z$,

(1) $x \leq x$, for any $x \quad$ (Reflexive),

(2) $x \leq y$ and $y \leq x$ implies $x=y$ (Antisymmetry),

(3) $x \leq y$ and $y \leq z$ implies $x \leq z \quad$ (Transitivity).

Definition 2.2 ( [1] ) A formal context is defined as a set structure $(G, M, I)$ consisting of sets $G$ and $M$ and a binary relation $I \subseteq G \times M$. The elements of $G$ and $M$ are called objects and attributes, respectively, and the relationship $g I m$ is read: the object $g$ has the attribute $m$. For a set of objects $A \subseteq G$, and a set of attributes $B \subseteq M$, $A^{*}$ is defined as the set of attributes common to the objects in $A, B^{*}$ is defined as the set of objects that posses all the attributes in $B$, that is,

$$
A^{*}=\{m \in M \mid g \operatorname{Im} \forall g \in A\} \quad B^{*}=\{g \in G \mid g \operatorname{Im} \forall m \in B\} .
$$

Definition 2.3 ( [1] ) A formal concept of the context $(G, M, I)$ is defined as a pair $(A, B)$ with $A \subseteq G$, $B \subseteq M$ and $A^{*}=B, B^{*}=A$. The set $A$ is called the extent and $B$ the intent of the concept $(A, B)$.

Example 2.1 A formal context and its Hasse diagram of the concept lattice are depicted as Table 1 and Fig. 1,

Table 1. A formal context $(G, M, I)$

\begin{tabular}{lllllll}
\hline & $a$ & $b$ & $c$ & $d$ & $e$ \\
\hline 1 & 1 & 1 & 0 & 0 & 1 \\
2 & 1 & 0 & 0 & 0 & 1 \\
3 & 0 & 1 & 1 & 1 & 0 \\
4 & 0 & 0 & 1 & 0 & 1 \\
\hline & & &
\end{tabular}

Fig. 1 Hasse diagram of concept lattice
Definition 2.4 ( $[16,17])$ Let $(L, \wedge, \vee, O, I)$ be a bounded lattice with an order-reversing involution'. $I$ and $O$ are the greatest and the smallest element of $L$, respectively. $\rightarrow: L \times L \rightarrow L$ is a mapping. If for any $x, y, z \in L$, the following conditions hold:

(1) $x \rightarrow(y \rightarrow z)=y \rightarrow(x \rightarrow z)$;

(2) $x \rightarrow x=I$;

(3) $x \rightarrow y=y^{\prime} \rightarrow x^{\prime}$;

(4) $x \rightarrow y=y \rightarrow x=I$ implies $x=y$;

(5) $(x \rightarrow y) \rightarrow y=(y \rightarrow x) \rightarrow x$;

(6). $(x \vee y) \rightarrow z=(x \rightarrow z) \wedge(y \rightarrow z)$;

(7). $(x \wedge y) \rightarrow z=(x \rightarrow z) \vee(y \rightarrow z)$.

Then $\left(L, \wedge, \vee,{ }^{\prime}, \rightarrow, O, I\right)$ is called a lattice implication algebra.

Example 2.2 Let $L_{6}=\{O, a, b, c, d, I\}$ be a partial ordered set depicted as Fig.2, the operations $\wedge$ and $\vee$ are defined as:

$$
\begin{gathered}
x \vee y=(x \rightarrow y) \rightarrow y, \\
x \wedge y=\left(\left(x^{\prime} \rightarrow y^{\prime}\right) \rightarrow y^{\prime}\right)^{\prime} .
\end{gathered}
$$

and $\rightarrow$ is defined as Table 2, and ' is defined as:

$$
I^{\prime}=O, O^{\prime}=I, a^{\prime}=c, c^{\prime}=a, b^{\prime}=d, d^{\prime}=b .
$$

Then $\left(L_{6}, \wedge, \vee{ }^{\prime}, \rightarrow, O, I\right)$ is a lattice implication algebra.

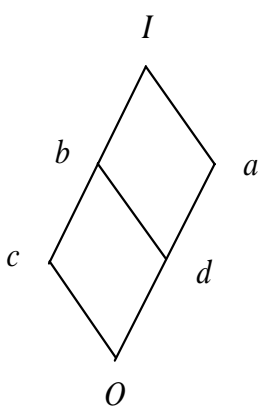

Fig. 2 Hasse diagram of $L_{6}=\{O, a, b, c, d, I\}$

Table 2. Implication operation of $L_{6}=\{O, a, b, c, d, I\}$

\begin{tabular}{lllllll}
\hline$\rightarrow$ & $O$ & $a$ & $b$ & $c$ & $d$ & $I$ \\
\hline$O$ & $I$ & $I$ & $I$ & $I$ & $I$ & $I$ \\
$a$ & $c$ & $I$ & $b$ & $c$ & $b$ & $I$ \\
$b$ & $d$ & $a$ & $I$ & $b$ & $a$ & $I$ \\
$c$ & $a$ & $a$ & $I$ & $I$ & $a$ & $I$ \\
$d$ & $b$ & $I$ & $I$ & $b$ & $I$ & $I$ \\
$I$ & $O$ & $a$ & $b$ & $c$ & $d$ & $I$ \\
\hline
\end{tabular}

$B C K$-algebra, $M V$-algebra, $M T L$-algebra, residuated lattice, $R_{0}$-algebra, and lattice implication algebra are all related to logic. The following theorems are mainly 
devoted to the discussion of relations between lattice implication algebra and the other algebras.

Theorem 2.1 Let $\left(L, \wedge, \vee{ }^{\prime}, \rightarrow, O, I\right)$ be a lattice implication algebra, if define $x \otimes y=\left(x \rightarrow y^{\prime}\right)^{\prime}$, then $(L, \otimes, \rightarrow)$ is a residuated lattice.

Theorem 2.2 Let $\left(L, \wedge, \vee,{ }^{\prime}, \rightarrow, O, I\right)$ be a lattice implication algebra. If define $x * y=y \rightarrow x$ for any $x, y \in L$,

then $(L, *, \theta)$ is a bounded commutative BCK-algebra with $e=O, \theta=I$.

if $\left(x^{\prime}\right)^{\prime}=x$ and $(x \rightarrow y) \rightarrow y=(y \rightarrow x) \rightarrow x \quad$, then $\left(L, \wedge, \vee{ }^{\prime}, \rightarrow, O, I\right)$ is a lattice implication algebra.

Theorem 2.3 Let $\left(L, \wedge, \vee{ }^{\prime}, \rightarrow, O, I\right)$ be a lattice implication algebra, define operations $*,+$ and $\cdot$ on $L$ as follows: for any $x, y \in L$,

$$
\begin{gathered}
x^{*}=x^{\prime} ; \\
x+y=x^{\prime} \rightarrow y ; \\
x \cdot y=\left(x \rightarrow y^{\prime}\right)^{\prime},
\end{gathered}
$$

then $(L,+, \cdot, *, O, I)$ is an $M V$-algebra.

Theorem 2.4 Let $(L,+, \cdot, *, O, I)$ be an $M V$-algebra, define a binary operation $\rightarrow$ and a unary operation 'on $L$ as follows: for any $x, y \in L$,

$$
\begin{aligned}
x \rightarrow y & =x^{*}+y ; \\
x^{\prime} & =x^{*},
\end{aligned}
$$

then $\left(L, \wedge, \vee,^{\prime}, \rightarrow, O, I\right)$ is a lattice implication algebra.

Theorem 2.5 Let $\left(L, \wedge, \vee,{ }^{\prime}, \rightarrow, O, I\right)$ be a lattice implication algebra, for any $x, y \in L$, if define $x \otimes y=\left(x \rightarrow y^{\prime}\right)^{\prime} \quad, \quad$ then $\quad(L, \vee, \wedge, \otimes, \rightarrow, O, I) \quad$ is $\quad$ a MTL-algebra.

Theorem 2.6 Let $(L, \vee, \wedge, \otimes, \rightarrow, O, I)$ is a $M T L$-algebra, for any $x \in L$ define:

$$
x^{\prime}=x \rightarrow O,
$$

if $\left(x^{\prime}\right)^{\prime}=x$ and $\quad(x \rightarrow y) \rightarrow y=(y \rightarrow x) \rightarrow x \quad$, then $\left(L, \wedge, \vee{ }^{\prime}, \rightarrow, O, I\right)$ is a lattice implication algebra.

Theorem 2.7 Let $\left(L, \wedge, \vee,{ }^{\prime}, \rightarrow, O, I\right)$ be a lattice implication algebra, for any $x, y \in L$, if $(x \rightarrow y) \vee\left((x \rightarrow y) \rightarrow\left(x^{\prime} \vee y\right)\right)=I \quad, \quad$ then $\left(L, \wedge, \vee,{ }^{\prime}, \rightarrow, O, I\right)$ is a a $R_{0}$-algebra.
Theorem 2.8 Let $(L, \vee, \wedge, \otimes, \rightarrow, O, I)$ is a $R_{0}$-algebra, for any $x, y \in L, \quad$ if $(x \rightarrow y) \rightarrow y=(y \rightarrow x) \rightarrow x$, then $\left(L, \wedge, \vee,{ }^{\prime}, \rightarrow, O, I\right)$ is a lattice implication algebra.

\section{Lattice-valued concept lattice}

Main theorem of L-concept lattice has two versions, the first one deals with the ordinary partial order on formal concepts. The second one deals with a fuzzy order on formal concepts. The L-Fuzzy concept theory has developed in $[18,19]$, which selects $L$ a support set of some structure $L=\langle L, \cdots\rangle$ as the scale of truth degrees. This paper selects a lattice implication algebra as the values range to construct the lattice-valued concept lattice. Based on the logical foundation, we can deal with not only the fuzziness and incomparability associated with the object itself, but also the uncertainty involved within the course of the object being processed as well. It is totally different from the fuzzy concept lattice. Its ideological core is to construct a lattice-valued fuzzy relation between objects and attributes. In this section, the definition of the lattice-valued concept lattice and its properties are presented.

Definition 3.1 A four-tuple $K=\left(G, M, L_{n}, \tilde{I}\right)$ is called a lattice-valued formal context, where $G=\left\{g_{1}, g_{2}, \cdots, g_{r}\right\}$ is the set of objects, $M=\left\{m_{1}, m_{2}, \cdots, m_{s}\right\}$ is the set of attributes, $L_{n}$ is an $n$-ary lattice implication algebra, $\tilde{I}$ is a relation between $G$ and $M$, i.e., $\tilde{I}: G \times M \rightarrow L_{n}$.

Similar to the definition of $L$-fuzzy set in [18], we have the following instructions:

Let $G$ be a non-empty set of object and $\left(L_{n}, \vee, \wedge,{ }^{\prime}, \rightarrow\right)$ an $n$-ary lattice implication algebra. Denote the set of all the $L_{n}$-fuzzy subsets on $G$ as $L_{n}^{G}$, $\forall A_{1}, A_{2} \in L_{n}^{G}, A_{1} \subseteq A_{2} \Leftrightarrow A_{1}(g) \leq A_{2}(g)$, for all $g \in G$, then $\left(L_{n}^{G}, \subseteq\right)$ is a partial ordered set.

Let $M$ be a non-empty set of attribute and $\left(L_{n}, \vee, \wedge,{ }^{\prime}, \rightarrow\right)$ an $n$-ary lattice implication algebra. Denote the set of all the $L_{n}$-fuzzy subsets on $M$ as $L_{n}^{M}$, $\forall B_{1}, B_{2} \in L_{n}^{M}, B_{1} \subseteq B_{2} \Leftrightarrow B_{1}(m) \leq B_{2}(m)$, for all $m \in M$, 
then $\left(L_{n}^{M}, \subseteq\right)$ is a partial ordered set.

According to [20-22], we can give the following theorem. The form of the two mappings in Theorem 3.1 is the same with the one in [20-22], but the operators are derived from the lattice implication algebra, which is not the same.

Theorem 3.1 Let $K=\left(G, M, L_{n}, \tilde{I}\right)$ be a lattice-valued formal context, $L_{n}$ be an $n$-ary lattice implication algebra, $f, h$ are two mappings between $L_{n}^{G}$ and $L_{n}^{M}$ defined as,

$$
\begin{gathered}
f: L_{n}^{G} \rightarrow L_{n}^{M} \\
f(A)(m)=\wedge_{g \in G}^{\wedge}(A(g) \rightarrow \tilde{I}(g, m)) \\
h: L_{n}^{M} \rightarrow L_{n}^{G} \\
h(B)(g)=\underset{m \in M}{\wedge}(B(m) \rightarrow \tilde{I}(g, m))
\end{gathered}
$$

Then $(f, h)$ is a Galois connection based on the lattice implication algebra.

Proof. For any $g \in G$,

$$
\begin{aligned}
A \subseteq h(B) & \Leftrightarrow A(g) \leq \underset{m \in M}{\wedge}(B(m) \rightarrow \tilde{I}(g, m)) \\
& \Leftrightarrow A(g) \leq B(m) \rightarrow \tilde{I}(g, m) \\
& \Leftrightarrow A(g) \rightarrow(B(m) \rightarrow \tilde{I}(g, m))=I \\
& \Leftrightarrow B(m) \rightarrow(A(g) \rightarrow \tilde{I}(g, m))=I \\
& \Leftrightarrow B(m) \leq A(g) \rightarrow \tilde{I}(g, m) \\
& \Leftrightarrow B(m) \leq \underset{g \in G}{\wedge}(A(g) \rightarrow \tilde{I}(g, m)) \\
& \Leftrightarrow B \subseteq f(A) .
\end{aligned}
$$

So $(f, h)$ is a Galois connection based on the lattice implication algebra.

Definition 3.2 A lattice-valued formal concept of $K=\left(G, M, L_{n}, \tilde{I}\right)$ is defined as a pair $(A, B)$ with $A \in L_{n}^{G}, \quad B \in L_{n}^{M}$ and $f(A)=B, h(B)=A$. For any $A_{1}, A_{2} \in L_{n}^{G} \quad, \quad B_{1}, B_{2} \in L_{n}^{M} \quad, \quad$ define $\left(A_{1}, B_{1}\right) \leq\left(A_{2}, B_{2}\right) \Leftrightarrow A_{1} \subseteq A_{2}\left(\right.$ or $\left.B_{2} \subseteq B_{1}\right)$ and the set $L(K)=\{(A, B) \mid f(A)=B, h(B)=A\} \quad$ is called the lattice-valued concept lattice.

Example 3.1 Let us consider the lattice-valued formal context $\left(G, M, L_{6}, \tilde{I}\right)$ depicted in Table 3 , where $G=\left\{g_{1}, g_{2}\right\}, M=\left\{m_{1}, m_{2}, m_{3}, m_{4}\right\}$, the attribute values are some linguistic values, such as: best, better, somewhat good, worst, worse, somewhat bad.

Table 3. A lattice-valued formal context $\left(G, M, L_{6}, \tilde{I}\right)$

\begin{tabular}{lcccc}
\hline$\tilde{I}$ & $m_{1}$ & $m_{2}$ & $m_{3}$ & $m_{4}$ \\
\hline$a_{1}$ & somewhat bad & worse & somewhat gond better \\
$g_{2}$ & worst & better & best & somewhat good
\end{tabular}

For the linguistic values in Table 3, we denote: $I=$ best, $O=$ worst, $a=$ somewhat bad, $b=$ better, $c=$ somewhat good, $d=$ worse. Then Table 3 can be written as the following Table 4. And in these linguistic values, there exist some incomparable values, so they can be established into a lattice implication algebra $L_{6}$, whose Hasse diagram is depicted in Fig 2:

Table 4. A lattice-valued formal context

\begin{tabular}{ccccc}
\hline$\tilde{I}$ & $m_{1}$ & $m_{2}$ & $m_{3}$ & $m_{4}$ \\
\hline$a_{1}$ & $a$ & $d$ & $c$ & $b$ \\
$g_{2}$ & $O$ & $b$ & $I$ & $c$ \\
\hline
\end{tabular}

Theorem 3.2 Let $K=\left(G, M, L_{n}, \tilde{I}\right)$ be a lattice-valued formal context, and $(f, h)$ the Galois connection, for any $A_{1}, A_{2}, A \in L_{n}^{G}, B_{1}, B_{2}, B \in L_{n}^{M}$, there are the following properties:

(1) $A_{1} \subseteq A_{2} \Rightarrow f\left(A_{2}\right) \subseteq f\left(A_{1}\right)$,

$B_{1} \subseteq B_{2} \Rightarrow h\left(B_{2}\right) \subseteq h\left(B_{1}\right)$;

(2) $A \subseteq h f(A), B \subseteq f h(B)$;

(3) $f(A)=f h f(A), g(B)=h f h(B)$;

Proof. (1) For any $A_{1}, A_{2} \in L_{n}^{G}, g \in G, m \in M$ $A_{1} \subseteq A_{2}$

$\Rightarrow A_{1}(g) \leq A_{2}(g)$

$\Rightarrow A_{2}(g) \rightarrow \tilde{I}(g, m) \leq A_{1}(g) \rightarrow \tilde{I}(g, m)$

$\Rightarrow \underset{g \in G}{\wedge}\left(A_{2}(g) \rightarrow \tilde{I}(g, m)\right) \leq \underset{g \in G}{\wedge}\left(A_{1}(g) \rightarrow \tilde{I}(g, m)\right)$

$\Rightarrow f\left(A_{2}\right)(m) \leq f\left(A_{1}\right)(m)$, for any $m \in M$

So, $A_{1} \subseteq A_{2} \Rightarrow f\left(A_{2}\right) \subseteq f\left(A_{1}\right)$;

$B_{1} \subseteq B_{2} \Rightarrow h\left(B_{2}\right) \subseteq h\left(B_{1}\right)$ can be proved similarly.

(2) For any $A \in L_{n}^{G}$, and $\forall g_{i} \in G$,

$h f(A)\left(g_{i}\right)$

$=\underset{m_{j} \in M}{\wedge}\left(f(A)\left(m_{j}\right) \rightarrow \tilde{I}\left(g_{i}, m_{j}\right)\right)$ 


$$
\begin{aligned}
& =\underset{m_{j} \in M}{\wedge}\left(\underset{g_{k} \in G}{\wedge}\left(A\left(g_{k}\right) \rightarrow \tilde{I}\left(g_{k}, m_{j}\right)\right) \rightarrow \tilde{I}\left(g_{i}, m_{j}\right)\right) \\
& =\underset{m_{j} \in M}{\wedge} \underset{g_{k} \in G}{\vee}\left(\left(A\left(g_{k}\right) \rightarrow \tilde{I}\left(g_{k}, m_{j}\right)\right) \rightarrow \tilde{I}\left(g_{i}, m_{j}\right)\right) \\
& \geq A\left(g_{i}\right),
\end{aligned}
$$

So, $A \subseteq h f(A)$;

$B \subseteq f h(B)$ can be proved similarly.

(3) For any $A \in L_{n}^{G}$, firstly, by (1) and (2), $A \subseteq h f(A) \Rightarrow f h f(A) \subseteq f(A)$, secondly, $\forall m_{j} \in M$,

$$
\begin{aligned}
& \text { fhf }(A)\left(m_{j}\right) \\
& =\underset{g_{i} \in G}{\wedge}\left(h f(A)\left(g_{i}\right) \rightarrow \tilde{I}\left(g_{i}, m_{j}\right)\right) \\
& =\underset{g_{i} \in G}{\wedge}\left(\underset{m_{k} \in M}{\wedge}\left(f(A)\left(m_{k}\right) \rightarrow \tilde{I}\left(g_{i}, m_{k}\right)\right) \rightarrow \tilde{I}\left(g_{i}, m_{j}\right)\right) \\
& =\underset{g_{i} \in G}{\wedge} \underset{m_{k} \in M}{\vee}\left(\left(f(A)\left(m_{k}\right) \rightarrow \tilde{I}\left(g_{i}, m_{k}\right)\right) \rightarrow \tilde{I}\left(g_{i}, m_{j}\right)\right) \\
& \geq f(A)\left(m_{j}\right) \text {, }
\end{aligned}
$$

it follows that, $f(A) \subseteq f h f(A)$.

So, $f(A)=f h f(A)$;

$h(B)=h f h(B)$ can be proved similarly.

\section{A combination algorithm of multiple lattice-valued concept lattices}

Concept lattice provide a theoretical framework for the design and discovery of concept hierarchies from relational information systems and the structure of every concept lattice is corresponding one by one with its formal context, so the distributed treatment of concept lattices certainly relates to some operations such as the decomposition and combination of formal context.

\subsection{Theoretical foundation for the combination operation}

Definition 4.1 Let $K_{1}=\left(G_{1}, M_{1}, L_{n}, \tilde{I}_{1}\right) \quad$ and $K_{2}=\left(G_{2}, M_{2}, L_{n}, \tilde{I}_{2}\right)$ be lattice-valued formal contexts. If $G_{1}=G_{2}, M_{1}=M_{2}, \tilde{I}_{1} \neq \tilde{I}_{2}$. Then $K_{1}$ and $K_{2}$ are called homotypic lattice-valued formal contexts and $L\left(K_{1}\right)$ and $L\left(K_{2}\right)$ are called homotypic lattice-valued concept lattices.

Definition 4.2 Let $K=\left(G, M, L_{n}, \tilde{I}\right), K_{1}=\left(G, M, L_{n}, \tilde{I}_{1}\right)$ and $K_{2}=\left(G, M, L_{n}, \tilde{I}_{2}\right)$ be homotypic lattice-valued formal contexts. If $\tilde{I}=\tilde{I}_{1} \wedge \tilde{I}_{2}$, i.e., $\forall g \in G, m \in M$, $\tilde{I}(g, m)=\tilde{I}_{1}(g, m) \wedge \tilde{I}_{2}(g, m)$. Then $K$ is called a combined formal context of $K_{1}$ and $K_{2}$, denoted as $K=K_{1} \wedge K_{2}$.

Definition 4.3 Let $K=\left(G, M, L_{n}, \tilde{I}\right)$ be a lattice-valued formal context, and $L(K)$ be the lattice-valued concept lattice. $\forall C_{i}=\left(A_{i}, B_{i}\right), C_{j}=\left(A_{j}, B_{j}\right) \in L(K)$,

(1) $C_{i}$ is said to be more than $C_{j}$, denoted as $C_{i}>C_{j}$, if $A_{i} \supset A_{j}$ (or equivalent $B_{j} \supset B_{i}$ );

(2) $C_{i}$ is said to be incomparable with $C_{j}$, denoted as $C_{i} \approx C_{j}$, if $A_{i} \approx A_{j}$ (or equivalent $B_{i} \approx B_{j}$ ).

Definition 4.4 Let $\left(L_{n}^{G}, \subseteq\right)$ be a partial ordered set, for any $A_{i}, A_{j} \in L_{n}^{G}, A_{j}$ is called a covering object set of $A_{i}$, denoted by $A_{i} \prec A_{j}$, if $A_{i} \subseteq A_{j}$ and there no $A_{x} \in L_{n}^{G}$ exists such that $A_{i} \subset A_{x} \subset A_{j}$.

Definition 4.5 Let $\left(L_{n}^{M}, \subseteq\right)$ be a partial ordered set, for any $B_{i}, B_{j} \in L_{n}^{M}, B_{j}$ is called a covering attribute set of $B_{i}$, denoted by $B_{i} \prec B_{j}$, if $B_{i} \subseteq B_{j}$ and there no $B_{y} \in L_{n}^{M}$ exists such that $B_{i} \subset B_{y} \subset B_{j}$.

Theorem 4.1 Let $K=\left(G, M, L_{n}, \tilde{I}\right)$ be a lattice-valued formal context, and $L(K)$ the lattice-valued concept lattice, $\forall C=(A, B) \in L(K)$,

(1)If $\exists A_{i} \prec A$, s.t., $\left(A_{i}, f\left(A_{i}\right)\right) \in L(K)$, then $B \prec f\left(A_{i}\right)$;

(2)If $\exists A_{i} \prec A \quad$, s.t., $\quad\left(A_{i}, f\left(A_{i}\right)\right) \notin L(K) \quad$, then $A_{i} \prec h\left(f\left(A_{i}\right)\right)$.

Proof. (1) For any $(A, B),\left(A_{i}, f\left(A_{i}\right)\right) \in L(K)$, $A_{i} \prec A \Rightarrow A_{i} \subset A \Rightarrow f(A) \subset f\left(A_{i}\right)$, i.e., $B \subset f\left(A_{i}\right)$; Suppose that $\exists B_{y} \in L_{n}^{M}$, s.t., $B \subset B_{y} \subset f\left(A_{i}\right)$, by Theorem 3.2 (1), we can get $h\left(f\left(A_{i}\right)\right) \subset h\left(B_{y}\right) \subset h(B)$, i.e., $A_{i} \subset h\left(B_{y}\right) \subset A$, this is in contradiction with $A_{i} \prec A$, so $B \prec f\left(A_{i}\right)$.

(2) By the definition of Galois connection $(f, h)$ on the formal context, we get

$$
h\left(f\left(A_{i}\right)\right)(g)
$$




$$
\begin{aligned}
& =\underset{m \in M}{\wedge}\left(f\left(A_{i}\right)(m) \rightarrow \tilde{I}(g, m)\right) \\
& =\underset{m \in M}{\wedge}\left(\underset{g \in G}{\wedge}\left(A_{i}(g) \rightarrow \tilde{I}(g, m)\right) \rightarrow \tilde{I}(g, m)\right) \\
& =\underset{m \in M}{\vee} \underset{g \in G}{\vee}\left(\left(A_{i}(g) \rightarrow \tilde{I}(g, m)\right) \rightarrow \tilde{I}(g, m)\right) \\
& =\underset{m \in M}{\vee}\left(A_{i \in G}(g) \vee \tilde{I}(g, m)\right) \\
& =\underset{g \in G}{\vee}\left(A_{i}(g) \vee(\underset{m \in M}{\wedge} \tilde{I}(g, m))\right) \\
& >A_{i}(g)
\end{aligned}
$$

That is to say, $A_{i} \subset h\left(f\left(A_{i}\right)\right)$.

Suppose that $\exists A_{x} \in L_{n}^{G}$, s.t., $A_{i} \subset A_{x} \subset h\left(f\left(A_{i}\right)\right)$, by theorem 3.2 (1), we can get $f\left(h\left(f\left(A_{i}\right)\right)\right) \subseteq f\left(A_{x}\right) \subseteq f\left(A_{i}\right)$, and further according to theorem 3.2 (3), we can get $f\left(A_{i}\right) \subseteq f\left(A_{x}\right) \subseteq f\left(A_{i}\right)$, i.e., $f\left(A_{i}\right)=f\left(A_{x}\right)$, then $A_{i}=A_{x}$, so, $A_{i} \prec h\left(f\left(A_{i}\right)\right)$.

Theorem 4.2 Let $K=\left(G, M, L_{n}, \tilde{I}\right)$ be a lattice-valued formal context, and $L(K)$ the lattice-valued concept lattice, if $\forall\left(A_{i}, f\left(A_{i}\right)\right) \notin L(K)$, then $\exists(A, B) \in L(K)$ and $A_{i} \prec A$, s.t., $f\left(A_{i}\right)=B$.

Proof. $\left(A_{i}, f\left(A_{i}\right)\right) \notin L(K) \Rightarrow\left(h\left(f\left(A_{i}\right)\right), f\left(A_{i}\right)\right) \in L(K)$ by Theorem 4.1, and $A_{i} \prec h\left(f\left(A_{i}\right)\right)$, so $\exists A=h\left(f\left(A_{i}\right)\right)$, s.t., $\left(A, f\left(A_{i}\right)\right) \in L(K)$, i.e., $f\left(A_{i}\right)=B$.

Definition 4.6 Let $K_{1}=\left(G, M, L_{n}, \tilde{I}_{1}\right) \quad$ and $K_{2}=\left(G, M, L_{n}, \tilde{I}_{2}\right)$ be lattice-valued homotypic formal contexts, $\forall C_{i}=\left(A_{i}, B_{i}\right) \in L\left(K_{1}\right), C_{j}=\left(A_{j}, B_{j}\right) \in L\left(K_{2}\right)$,

(1) $C_{i}$ is said to be homotypicly equal to $C_{j}$, denoted as $C_{i} \cong C_{j}$, if $A_{i}=A_{j}$;

(2) $C_{i}$ is said to be homotypicly more than $C_{j}$, denoted as $C_{i} \tilde{>} C_{j}$, if $A_{i} \supset A_{j}$;

(3) $C_{i}$ is said to be homotypicly covering $C_{j}$, denoted as $C_{j} \approx C_{i}$, if $A_{j} \prec A_{i}$;

(4) $C_{i}$ is said to be homotypicly incomparable with $C_{j}$, denoted as $C_{i} \approx C_{j}$, if $A_{i} \approx A_{j}$;

(5) $C=(A, B)$ is said to be combined formal concept of $C_{i}$ and $C_{j}$, denoted as $C=C_{i} \wedge C_{j}$, if $A=A_{i} \wedge A_{j}$, $B=B_{i} \wedge B_{j}$.
Remark. By (1) and (2) of Definition 4.6, we will get the conclusion: $C_{i} \tilde{\geq} C_{j}$, if $A_{i} \supseteq A_{j}$.

Definition $\quad 4.7 \quad$ Let $K=\left(G, M, L_{n}, \tilde{I}\right) \quad$, $K_{1}=\left(G, M, L_{n}, \tilde{I}_{1}\right) \quad$ and $\quad K_{2}=\left(G, M, L_{n}, \tilde{I}_{2}\right) \quad$ be lattice-valued homotypic formal contexts. $L(K)$, $L\left(K_{1}\right), L\left(K_{2}\right)$ are the lattice-valued homotypic concept lattices. $L(K)$ is said to be a combined concept lattice of $L\left(K_{1}\right)$ and $L\left(K_{2}\right)$, denoted by $L(K)=L\left(K_{1}\right) \wedge L\left(K_{2}\right)$, if $\forall C \in L(K), \exists C_{1} \in L\left(K_{1}\right)$ and $C_{2} \in L\left(K_{2}\right)$, s.t., $C=C_{1} \tilde{\wedge} C_{2}$, and satisfies $C_{i} \tilde{\succ} C$ or $C_{i} \cong C, i=1,2$.

Definition 4.8 Let $K=\left(G, M, L_{n}, \tilde{I}_{1}\right) \quad$ and $K_{2}=\left(G, M, L_{n}, \tilde{I}_{2}\right)$ be lattice-valued homotypic formal contexts. $K=\left(G, M, L_{n}, \tilde{I}\right)$ is the combined formal context of $K_{1}$ and $K_{2}$. Let $L(K), L\left(K_{1}\right), L\left(K_{2}\right)$ be the lattice-valued homotypic concept lattices and $L(K)=L\left(K_{1}\right) \wedge L\left(K_{2}\right) \quad, \quad \forall C=(A, B) \in L(K) \quad$ ， $\exists C_{1}=\left(A_{1}, B_{1}\right) \in L\left(K_{1}\right) \quad$ and $\quad C_{2}=\left(A_{2}, B_{2}\right) \in L\left(K_{2}\right)$, s.t., $C=C_{1} \tilde{\wedge} C_{2}$.

(1) $C$ is said to be the intent updating fuzzy concept of $C_{1}$ and $C_{2}$, if $C \cong C_{1}$ or $C \cong C_{2}$ or $C \cong C_{1} \cong C_{2}$;

(2) $C$ is said to be the newly added fuzzy concept of $C_{1}$ and $C_{2}$, if $C \tilde{\prec} C_{1}$ and $C \prec C_{2}$.

Theorem $\quad 4.3 \quad$ Let $\quad K_{1}=\left(G, M, L_{n}, \tilde{I}_{1}\right) \quad$ and $K_{2}=\left(G, M, L_{n}, \tilde{I}_{2}\right)$ be lattice-valued homotypic formal contexts. $L\left(K_{1}\right), L\left(K_{2}\right)$ are the lattice-valued homotypic concept lattices. Then $L\left(K_{1}\right) \wedge L\left(K_{2}\right)=L\left(K_{1} \wedge K_{2}\right)$.

Proof. Suppose that $\left(f_{1}, h_{1}\right),\left(f_{2}, h_{2}\right)$ and $(f, h)$ are the Galois connections on $K_{1}, K_{2}$ and $K_{1} \wedge K_{2}$, respectively. $\forall C=(A, B) \in L\left(K_{1}\right) \wedge L\left(K_{2}\right)$, $\exists C_{1}=\left(A_{1}, B_{1}\right) \in L\left(K_{1}\right), C_{2}=\left(A_{2}, B_{2}\right) \in L\left(K_{2}\right)$, s.t., $C=C_{1} \wedge C_{2}$, it follows that $A=A_{1} \wedge A_{2}, B=B_{1} \wedge B_{2}$, then $A \tilde{\prec} A_{1}$ and $A \tilde{\prec} A_{2}$, since $(A, B) \in L\left(K_{1}\right) \wedge L\left(K_{2}\right)$, we can get $(A, B) \notin L\left(K_{1}\right)$ and $(A, B) \notin L\left(K_{2}\right)$. So $f_{1}(A)=B_{1}$ in the fuzzy context $K_{1}$ and $f_{2}(A)=B_{2}$ in the fuzzy context $K_{2}$ can be get by Theorem 4.2. In addition,

$$
\begin{aligned}
B(m) & =\left(B_{1} \wedge B_{2}\right)(m) \\
& =\left(f_{1}(A) \wedge f_{2}(A)\right)(m) \\
= & \underset{g \in G}{\wedge}\left(A(g) \rightarrow \tilde{I}_{1}(g, m)\right) \wedge \underset{g \in G}{\wedge}\left(A(g) \rightarrow \tilde{I}_{2}(g, m)\right)
\end{aligned}
$$




$$
\begin{aligned}
& =\bigwedge_{g \in G}\left(A(g) \rightarrow \tilde{I}_{1}(g, m) \wedge \tilde{I}_{2}(g, m)\right) \\
& =\underset{g \in G}{\wedge}(A(g) \rightarrow \tilde{I}(g, m)) \\
& =f(A)(m)
\end{aligned}
$$

Therefore, $C=(A, B) \in L\left(K_{1} \wedge K_{2}\right)$.

Conversely, suppose that $\forall C=(A, B) \in L\left(K_{1} \wedge K_{2}\right)$, then we can get $\exists C_{1}=\left(A_{1}, B_{1}\right) \in L\left(K_{1}\right)$, $C_{2}=\left(A_{2}, B_{2}\right) \in L\left(K_{2}\right)$ and satisfying $C \approx C_{1}$ or $C \cong C_{1}, C \approx C_{2}$ or $C \cong C_{2}$. It follows that, if $C \approx C_{1}$ and $C \approx C_{2}$, since $C \notin L\left(K_{1}\right)$ and $C \notin L\left(K_{2}\right)$, by Theorem 4.2, we get $f_{1}(A)=B_{1}$ in fuzzy context $K_{1}$ and $f_{2}(A)=B_{2}$ in fuzzy context $K_{2}$. In fuzzy context $K_{1} \wedge K_{2}$, for $m \in M$,

$$
\begin{aligned}
B(m) & =f(A)(m) \\
& =\underset{g \in G}{\wedge}(A(g) \rightarrow \tilde{I}(g, m)) \\
& =\underset{g \in G}{\wedge}\left(A(g) \rightarrow\left(\tilde{I}_{1}(g, m) \wedge \tilde{I}_{2}(g, m)\right)\right) \\
& =\underset{g \in G}{\wedge}\left(A(g) \rightarrow \tilde{I}_{1}(g, m)\right) \wedge \wedge_{g \in G}^{\wedge}\left(A(g) \rightarrow \tilde{I}_{2}(g, m)\right) \\
& =f_{1}(A)(m) \wedge f_{2}(A)(m) \\
& =B_{1}(m) \wedge B_{2}(m)
\end{aligned}
$$

Therefore, $B=B_{1} \wedge B_{2}$.

$$
\begin{aligned}
& \text { For } C_{1}=\left(A_{1}, B_{1}\right)=\left(h_{1}\left(f_{1}(A)\right), f_{1}(A)\right) \text {, } \\
& C_{2}=\left(A_{2}, B_{2}\right)=\left(h_{2}\left(f_{2}(A)\right), f_{2}(A)\right) \text { and } g \in G, \\
& \left(h_{1}\left(f_{1}(A)\right) \wedge h_{2}\left(f_{2}(A)\right)\right)(g) \\
& =\underset{m \in M}{\wedge}\left(f_{1}(A)(m) \rightarrow \tilde{I}_{1}(g, m)\right) \wedge \underset{m \in M}{\wedge}\left(f_{2}(A)(m) \rightarrow \tilde{I}_{2}(g, m)\right) \\
& =\underset{m \in M}{\wedge}\left(\underset{g \in G}{\vee}\left(A(g) \vee \tilde{I}_{1}(g, m)\right) \wedge \underset{g \in G}{\vee}\left(A(g) \vee \tilde{I}_{2}(g, m)\right)\right) \\
& =\underset{m \in M}{\vee} \underset{g \in G}{\vee}\left(\left(A(g) \vee \tilde{I}_{1}(g, m)\right) \wedge\left(A(g) \vee \tilde{I}_{2}(g, m)\right)\right) \\
& =\underset{m \in M}{\wedge} \underset{g \in G}{\vee}\left(A(g) \vee\left(\tilde{I}_{1}(g, m) \wedge \tilde{I}_{2}(g, m)\right)\right) \\
& =\underset{m \in M}{\wedge} \underset{g \in G}{\vee}(A(g) \vee \tilde{I}(g, m)) \\
& =\underset{m \in M}{\wedge}(\underset{g \in G}{\wedge}(A(g) \rightarrow \tilde{I}(g, m)) \rightarrow \tilde{I}(g, m)) \\
& =\wedge_{m \in M}(f(A)(m) \rightarrow \tilde{I}(g, m)) \\
& =h(f(A))(g) \\
& =A(g)
\end{aligned}
$$

Therefore, $A=A_{1} \wedge A_{2}$.

On the other hand, if $C_{1} \simeq C$ and $C_{2} \simeq C$, then $A=A_{1}=A_{2}$. It is obvious that $C=C_{1} \wedge C_{2}$. Hence $C=(A, B) \in L\left(K_{1}\right) \wedge L\left(K_{2}\right)$.
Corollary $4.1 \quad$ Let $K_{1}=\left(G_{1}, M_{1}, L_{n}, \tilde{I}_{1}\right)$, $K_{2}=\left(G_{2}, M_{2}, L_{n}, \tilde{I}_{2}\right), \cdots, K_{m}=\left(G_{m}, M_{m}, L_{n}, \tilde{I}_{m}\right)(m \geq 2)$ be lattice-valued homotypic formal contexts. $L\left(K_{1}\right)$, $L\left(K_{2}\right), \cdots, L\left(K_{m}\right)$ are the lattice-valued homotypic concept lattices. Then $L\left(K_{1}\right) \wedge L\left(K_{2}\right) \wedge \cdots \wedge L\left(K_{m}\right)=L\left(K_{1} \wedge K_{2} \wedge \cdots \wedge K_{m}\right)$.

\subsection{Algorithm description and analysis}

The basic idea of this combination algorithm is to construct the formal concepts of every simple lattice-valued concept lattice and combine them according to the combination operation definition.

Algorithm: A combination algorithm of lattice-valued concept lattice

Calculate the formal concepts $C_{i j}=\left(A_{i j}, B_{i j}\right)$ of $K_{i}$, $i=1,2, \cdots, m ; j=1,2, \cdots,\left\|L\left(K_{i}\right)\right\| ;\left\|L\left(K_{i}\right)\right\|$ is the number of formal concepts of $L\left(K_{i}\right)$.

Input: the formal concepts of $K_{1}, K_{2}, \cdots, K_{m}(m \geq 2)$.

Output: the formal concepts of $K_{1} \wedge K_{2} \wedge \cdots \wedge K_{m} \triangleq K$

Begin

while $\left(K_{1}, K_{2}, \cdots, K_{m} \neq \Phi\right)$ do

for $i \leftarrow 1$ to $m$ do

$$
\text { for } j \leftarrow 1 \text { to }\left\|L\left(K_{i}\right)\right\| \text { do }
$$

$$
C_{i j}:=C_{i j}
$$

$$
\text { for } p \leftarrow 1 \text { to } m \text { do }
$$$$
\text { if } p \neq i \text { then }
$$

$$
\begin{gathered}
\text { for } q \leftarrow 1 \text { to }\left\|L\left(K_{p}\right)\right\| \text { do } \\
\text { if } C_{p q} \cong C_{i j} \text { then } \\
C_{i j}:=C_{i j} \wedge C_{p q}
\end{gathered}
$$

\section{endif;}

$$
\text { if } \begin{aligned}
C_{i j} & \approx C_{p q} \text { then } \\
C_{i j} & :=C_{i j} \wedge C_{p q}
\end{aligned}
$$

endif;

endfor;

\section{else}

endfor;

else

endfor;

endfor;

endfor;

end; 
The time complexity of creating concept lattices is the major factor in analyzing the complexity of the algorithm, and computing the formal concepts plays the key role in the whole process of constructing concept lattices. Suppose the lattice-valued formal context $K=\left(G, M, L_{n}, \tilde{I}\right)$, where $G=\left\{g_{1}, g_{2}, \cdots, g_{r}\right\}$, $M=\left\{m_{1}, m_{2}, \cdots, m_{s}\right\}, r, s$ and $n$ are the positive integers, the time complexity of computing the formal concepts is $O\left(2 r \times n^{s}\right)$.

$$
\text { If we let } \begin{aligned}
M= & \left\{m_{1}, m_{2}, \cdots, m_{s}\right\} \\
= & \left\{m_{1}, \cdots, m_{s_{1}}\right\} \bigcup\left\{m_{s_{1}+1}, \cdots, m_{s_{1}+s_{2}}\right\} \\
& \cup \cdots \cup\left\{m_{s_{p-1}+1}, \cdots, m_{s_{p-1}+s_{p}}\right\}
\end{aligned}
$$

where $s=s_{1}+s_{2}+\cdots+s_{p}, s_{1}, s_{2}, \cdots, s_{p}$ are also the positive integers, and the combination algorithm is executed on them, then the time complexity of calculating the formal concepts is $O\left(2 r \times\left(n^{s_{1}}+n^{s_{2}}+\cdots+n^{s_{p}}\right)\right) \quad$. Because of $s_{1}, s_{2}, \cdots, s_{p}<s$, we can get $n^{s_{1}}+n^{s_{2}}+\cdots+n^{s_{p}}<<n^{s}$, then $O\left(2 r \times\left(n^{s_{1}}+n^{s_{2}}+\cdots+n^{s_{p}}\right)\right)<<O\left(2 r \times n^{s}\right)$, that is to say, the complexity of this algorithm is significantly decreased.

Example 4.1 Let us consider the lattice-valued concept lattice $K=\left(G, M, L_{6}, \tilde{I}\right)$ depicted in Table 4 , which can be looked as the combined formal context of $K_{1}=\left(G, M, L_{6}, \tilde{I}_{1}\right)$ and $K_{2}=\left(G, M, L_{6}, \tilde{I}_{2}\right)$ depicted in Table 5 and Table 6 :

Table 5. A lattice-valued formal context $K_{1}=\left(G, M, L_{6}, \tilde{I}_{1}\right)$

\begin{tabular}{ccccc}
\hline$\tilde{I}$ & $m_{1}$ & $m_{2}$ & $m_{3}$ & $m_{4}$ \\
\hline$g_{1}$ & $a$ & $d$ & $I$ & $I$ \\
$g_{2}$ & $O$ & $b$ & $I$ & $I$ \\
\hline
\end{tabular}

Table 6. A lattice-valued formal context $K_{2}=\left(G, M, L_{6}, \tilde{I}_{2}\right)$

\begin{tabular}{ccccc}
\hline$\tilde{I}$ & $m_{1}$ & $m_{2}$ & $m_{3}$ & $m_{4}$ \\
\hline$g_{1}$ & $I$ & $I$ & $c$ & $b$ \\
$g_{2}$ & $I$ & $I$ & $I$ & $c$ \\
\hline
\end{tabular}

About Table 4, Table 5 and Table 6, we can get the following relations:

$G=G_{1}=G_{2}=\left\{g_{1}, g_{2}\right\}$,
$M=M_{1}=M_{2}=\left\{m_{1}, m_{2}, m_{3}, m_{4}\right\}$,

$\tilde{I}(m, g) \neq \tilde{I}_{1}(m, g) \neq \tilde{I}_{2}(m, g)$, so $K, K_{1}$ and $K_{2}$ are lattice-valued homotypic formal contexts and $K=K_{1} \wedge K_{2}$. Hence, the formal concepts of Table 4 can be computed by Table 5 and Table 6 .

The formal concepts of Table 5 and the Hasse diagram of $L\left(K_{1}\right)$ are as Fig 3, in which,

$\begin{array}{ll}C_{0}=(\{I, I\},\{O, d, I, I\}) & C_{1}=(\{a, I\},\{O, b, I, I\}) \\ C_{2}=(\{a, a\},\{c, b, I, I\}) & C_{3}=(\{I, b\},\{d, d, I, I\}) \\ C_{4}=(\{a, b\},\{d, b, I, I\}) & C_{5}=(\{b, b\},\{d, a, I, I\}) \\ C_{6}=(\{d, b\},\{d, I, I, I\}) & C_{7}=(\{I, c\},\{a, d, I, I\}) \\ C_{8}=(\{a, c\},\{a, b, I, I\}) & C_{9}=(\{b, c\},\{a, a, I, I\}) \\ C_{10}=(\{d, c\},\{a, I, I, I\}) & C_{11}=(\{a, d\},\{b, b, I, I\}) \\ C_{12}=(\{d, d\},\{b . I, I, I\}) & C_{13}=(\{a, O\},\{I, b, I, I\}) \\ C_{14}=(\{d, O\},\{I, I, I, I\}) & \end{array}$

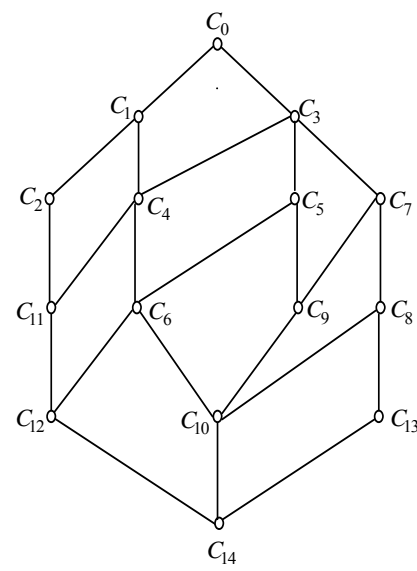

Fig. 3 Hasse diagram of $L\left(K_{1}\right)$

The formal concepts of Table 6 and the Hasse diagram of $L\left(K_{2}\right)$ are as Fig 4:

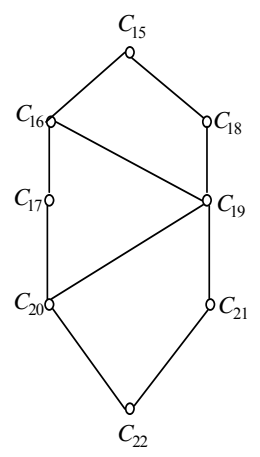

Fig. 4 Hasse diagram of $L\left(K_{2}\right)$

$$
C_{15}=(\{I, I\},\{I, I, c, c\}) \quad C_{16}=(\{b, I\},\{I, I, b, c\})
$$




$$
\begin{array}{ll}
C_{17}=(\{c, I\},\{I, I, I, c\}) & C_{18}=(\{I, b\},\{I, I, c, b\}) \\
C_{19}=(\{b, b\},\{I, I, b, b\}) & C_{20}=(\{c, b\},\{I, I, I, b\}) \\
C_{21}=(\{b, c\},\{I, I, b, I\}) & C_{22}=(\{c, c\},\{I, I, I, I\})
\end{array}
$$

For convenient computation, we combine the Fig. 3 with Fig. 4 as Fig. 5 according to the relations of formal concepts derived from the lattice-valued homotypic fuzzy contexts:

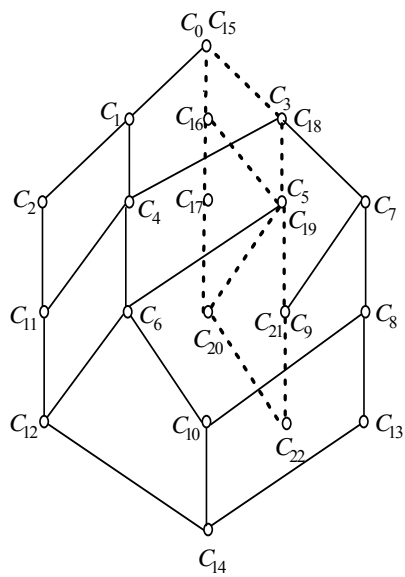

Fig. 5 Hasse diagram of the combination of $L\left(K_{1}\right)$ and $L\left(K_{2}\right)$

Through Fig. 5, we can compute the intent updating formal concepts and the newly added formal concepts according to the Definition 4.8. The formal concepts and its Hasse diagram of $L(K)$ are shown in Fig. 6:

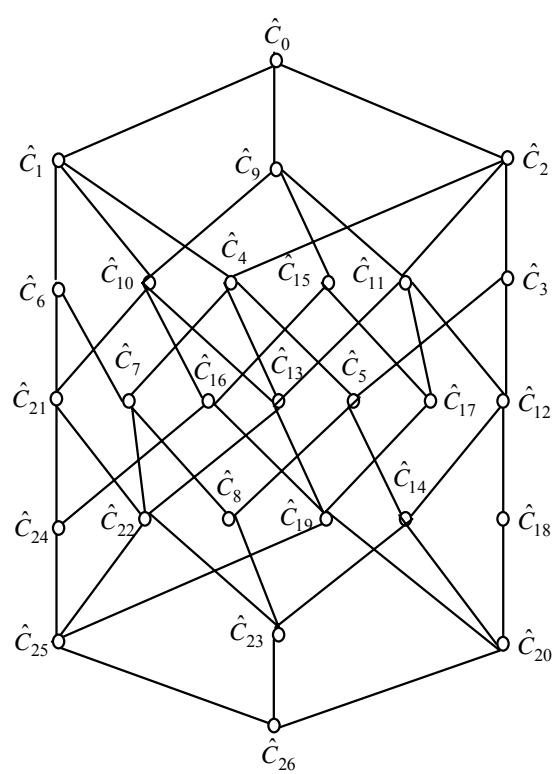

Fig. 6 Hasse diagram of $L(K)=L\left(K_{1}\right) \wedge L\left(K_{2}\right)$

$$
\hat{C}_{0}=C_{0} \wedge C_{15}=(\{I, I\},\{O, d, c, c\})
$$

$$
\begin{aligned}
& \hat{C}_{1}=C_{1} \wedge C_{15}=(\{a, I\},\{O, b, c, c\}) \\
& \hat{C}_{2}=C_{16} \wedge C_{0}=(\{b, I\},\{O, d, b, c\}) \\
& \hat{C}_{3}=C_{17} \wedge C_{0}=(\{c, I\},\{O, d, I, c\}) \\
& \hat{C}_{4}=C_{1} \wedge C_{16}=(\{d, I\},\{O, b, b, c\}) \\
& \hat{C}_{5}=C_{1} \wedge C_{17}=(\{O, I\},\{O, b, I, C\}) \\
& \hat{C}_{6}=C_{2} \wedge C_{15}=(\{a, a\},\{c, b, c, c\}) \\
& \hat{C}_{7}=C_{2} \wedge C_{16}=(\{d, a\},\{c, b, b, c\}) \\
& \hat{C}_{8}=C_{2} \wedge C_{17}=(\{O, a\},\{c, b, I, c\}) \\
& \hat{C}_{9}=C_{3} \wedge C_{18}=(\{I, b\},\{d, d, c, b\}) \\
& \hat{C}_{10}=C_{4} \wedge C_{18}=(\{a, b\},\{d, b, c, b\}) \\
& \hat{C}_{11}=C_{5} \wedge C_{19}=(\{b, b\},\{d, a, b, b\}) \\
& \hat{C}_{12}=C_{20} \wedge C_{5}=(\{c, b\},\{d, a, I, b\}) \\
& \hat{C}_{13}=C_{6} \wedge C_{19}=(\{d, b\},\{d, I, b, b\}) \\
& \hat{C}_{14}=C_{6} \wedge C_{20}=(\{O, b\},\{d, I, I, b\}) \\
& \hat{C}_{15}=C_{7} \wedge C_{18}=(\{I, c\},\{a, d, c, b\}) \\
& \hat{C}_{16}=C_{8} \wedge C_{18}=(\{a, c\},\{a, b, c, b\}) \\
& \hat{C}_{17}=C_{9} \wedge C_{21}=(\{b, c\},\{a, a, b, I\}) \\
& \hat{C}_{18}=C_{22} \wedge C_{9}=(\{c, c\},\{a, a, I, I\}) \\
& \hat{C}_{19}=C_{10} \wedge C_{21}=(\{d, c\},\{a, I, b, I\}) \\
& \hat{C}_{20}=C_{10} \wedge C_{22}=(\{O, c\},\{a, I, I, I\}) \\
& \hat{C}_{21}=C_{11} \wedge C_{18}=(\{a, d\},\{b, b, c, b\}) \\
& \hat{C}_{22}=C_{12} \wedge C_{19}=(\{d, d\},\{b . I, b, b\}) \\
& \hat{C}_{23}=C_{12} \wedge C_{20}=(\{O, d\},\{b, I, I, b\}) \\
& \hat{C}_{24}=C_{13} \wedge C_{18}=(\{a, O\},\{I, b, c, b\}) \\
& \hat{C}_{25}=C_{14} \wedge C_{21}=(\{d, O\},\{I, I, b, I\}) \\
& \hat{C}_{26}=C_{14} \wedge C_{22}=(\{O, O\},\{I, I, I, I\})
\end{aligned}
$$

\section{Conclusions}

For realizing the distributed computation and parallel processing of the lattice-valued fuzzy concept lattice, it is inevitable to research its combination algorithms. As the one of combination algorithms, this paper proposed a conjunction algorithm of multiple lattice-valued fuzzy concept lattices, which not only provides an effective method for constructing lattice-valued fuzzy concept lattice but also makes an important progress toward 
practical applications in decision-making. Concretely, we defined the homotypic lattice-valued fuzzy concept lattice and the conjunction fuzzy concept lattice as the preconditions and analyzed some relations between fuzzy concepts; successively, we gave some theorems to prove the isomorphism of conjunction theory and presented the conjunction algorithm of the lattice-valued fuzzy concept lattice. Obviously, before combining these simple lattice-valued fuzzy concept lattices, we should provide an appropriate decomposition method of the complex one, which will be our future research work.

\section{Acknowledgements}

Project was partially supported by the National Natural Science Foundation of P. R. China (Grant No. 71101049; 61175055) and Sichuan Key Technology Research and Development Program under (Grant No. 2011FZ0051).

\section{References}

[1] B. Ganter, R. Wille, Formal Concept Analysis: Mathematical Foundations, Springer, Berlin, Heidelberg, 1999.

[2] R. Wille, Restructuring lattice theory: An approach based on hierarchies of concepts. I: Rival I, ed.Ordered Sets. Dordrecht-Boston: Reidel, 445-470, 1982.

[3] R. Wille, Knowledge acquisition by methods of formal concept analysis, in: E. Diday (Ed.), Data Analysis, Learning Symbolic and Numeric Knowledge, Nova Science, NewYork, 1989.

[4] C. Carpineto, G. Romano, A lattice conceptual clustering system and its application to browsing retrieval, Machine Learning, 10: 95-122, 1996.

[5] D. Dubois, H. Prade: Possibility theory and formal concept analysis: Characterizing independent sub-contexts. Fuzzy Sets and Systems, 196: 4-16, 2012.

[6] J. Medina: Multi-adjoint property-oriented and object-oriented concept lattices. Inf. Sci., 190: 95-106, 2012.

[7] L. Yang, Y. Xu, Study of fuzzy concept lattice based on lattice-valued logic, Fuzzy Systems and Mathematics,
23(5): 15-20, 2009.

[8] L. Yang, Y. Xu, Construction Method of Lattice-Valued Fuzzy Concept Lattice Based on Matrix Implication Operation, Computer Science, 36(8): 264-267, 2009.

[9] L. Yang, Y. Xu, Decision making with uncertainty information based on lattice-valued fuzzy concept lattice, Journal of Universal Computer Sciences, 16(1): 159-177, 2010.

[10] L. Yang, Y. Xu, A decision method based on uncertainty reasoning of linguistic truth-valued concept lattice, International Journal of General Systems, 39(3): 235-253, 2010.

[11] L. Zhang, X.J. Shen, D.J. Han, et al. Vertical union algorithm of concept lattices based on synonymous concept, Computer Engineering and Applications, 43(2): 95-98, 2007.

[12] Y. Li, Z.T. Liu, L. Chen, X.H. Xu, W. Cheng, Horizontal union algorithm of multiple concept lattice, Acta Electronica Sinica, 32(11): 1849-1854, 2004.

[13] Z.T. Liu, L.S. Li, Q. Zhang, Research on a union algorithm of multiple concept lattices[C] RSFDGrC 2003, LNAI 2639. Berlin: Springer-Verlag Heidelberg, 533-540, $2003 .$.

[14] R. Belohlavek, "A note on variable threshold concept lattices: Threshold-based operators are reducible to classical concept-forming operators", Information Sciences, 177: 3186-3191, 2007.

[15] G. Birkhoff, Lattice Theory, in: American Mathematical Society Colloquium Publications XXV, American Mathematical Society, Providence, RI, 25,1967.

[16] Y. Xu, Lattice implication algebras, J. Southwest Jiaotong Univ., 289: 20-27, 1993 (in Chinese).

[17] Y. Xu, D. Ruan, K.Y. Oin, J. Liu, Lattice-Valued Logic-An Alternative Approach to Treat Fuzziness and Incomparability. Springer-Verlag, Berlin. 2003.

[18] J.A. Goguen, L-Fuzzy sets, Journal of Mathematical Analysis and Applications, 18(1): 145-174, 1967.

[19] J.A. Goguen, The logic of inexact concepts, Synthese, 19(3-4): 325-373, 1969.

[20] S. Pollandt, Fuzzy-begriffe: formale begriffs analyse unscharfer daten, Springer-Verlag, Berlin/Heidelberg, 
Li Yang, et. al

1997.

[21] R. Belohlávek Fuzzy concepts and conceptual structures: induced similarities, Joint Conference on Information Sciences, 179-182, 1998.

[22] R. Belohlavek, Snasel V., What is a fuzzy concept lattice, CLA, 34-45, 2005. 